\title{
Simulation Analysis of the Equipotential Surface of Regular-Shaped Charged Bodies
}

\author{
Jiahui Chen, Yingqiu Li, Ranran Li and Yi Jin* \\ Department of Physics, University of Jinan, 250022 Jinan, P.R. China \\ ${ }^{*}$ Corresponding author
}

Keywords: Equipotential surface, Regular-shaped charged bodies, Electromagnetics, MATLAB.

\begin{abstract}
The Potential distribution is an important physical quantity for describing the characteristics of charged objects in electromagnetic field. In order to discuss the characteristics of electric field strength and potential of charged objects more intuitively, the potential generated by various uniformly charged regular objects is calculated analytically, the distribution of equipotential points between two charged objects in three-dimensional space is discussed and the numerical simulation is carried out by using MATLAB.
\end{abstract}

\section{Introduction}

Electric field is widely used in daily life, engineering construction and scientific research. For example, applying an inhomogeneous electric field to the Ene-Silicon material can make the different regions of the material in different quantum phases, such as topological insulators and metals at the same time, which is easy to play an important role in the research and application of Spintronics[1].Under the action of electric field, laser dyes are doped chirally into liquid crystals to make liquid crystal lasers[2]. Dielectrics have electrothermal effects in electric fields, which can help to strengthen the convection process. Therefore, refrigeration devices are designed and manufactured by using the temperature change of dielectrics in the electric field[3]. People usually design different charged bodies according to actual needs. Electric field strength and electric potential are important physical quantities for investigating the design results. However, the electric field intensity is difficult to measure, so we usually consider the characteristics of charged bodies through electric potential or equipotential surface. Therefore, potential and equipotential surface have important practical value. In this paper, several common uniformly charged regular bodies are chosen and combined. The expressions of their field strength and potential are written according to the basic theory of Electromagnetics, and the equipotential surface shapes among charged objects are simulated and analyzed by MATLAB. Finally, the basic methods of solving space potential surfaces are summarized.

\section{Distribution of Equivalent Surface and Electrical Potential of Common Uniformly Charged Regular Bodies}

In the process of studying Electromagnetics, the commonly used methods to solve the electric field intensity are as follows: the element method based on the superposition principle of electric field, the Gauss' theorem, the symmetry method based on the symmetry of charged bodies, electric image method and so on[4]. In the following, the potential distribution of the most common uniformly charged regular bodies, like spherical surface and long straight wire are discussed.

The radius of the sphere is set to $R$, and the charge carried by the sphere is $q$. Because of the high symmetry of the sphere, the potential are obtained by using Gauss' theorem as follows:

$$
U=\frac{q}{4 \pi \varepsilon_{0} R}(r \leq R) \text { or } \frac{q}{4 \pi \varepsilon_{0} r}(r>R) .
$$

Where $r$ is the distance from the field point to coordinate origin, i.e., centre of sphere. The potential distribution in Eq. 1 when $r>R$ is plotted by MATLAB as shown in Fig. 1(a), which shows that the 
equipotential surface of the spherical surface is the concentric sphere. For simplification, $1 /\left(4 \pi \varepsilon_{0}\right)$ is taken as 1 in the plots hereafter. The electric field intensity is strong near the sphere, the equipotential surface is densely distributed. The electric field intensity is weak when far from the sphere and the equipotential surface is sparse.

Uniformly charged long straight wire is also common regular charged body. Setting the linear density of charge as $\lambda$ and the length of wire as 1 , the potential of long straight wire are obtained by superposition principle:

$$
U=\frac{\lambda}{4 \pi \varepsilon_{0}} \ln \frac{z+\frac{l}{2}+\sqrt{r^{2}+\left(z+\frac{l}{2}\right)^{2}}}{z-\frac{l}{2}+\sqrt{r^{2}+\left(z-\frac{l}{2}\right)^{2}}} .
$$

The potential and equipotential surface distributions are shown in Fig. 1(b) and (c) separately. It can be seen that the distributions of potential and equipotential surface are both related to the shape of charged bodies. The distribution of equipotential surface is ellipsoid around the long straight wire. It is dense near the wire and sparse away from the wire.

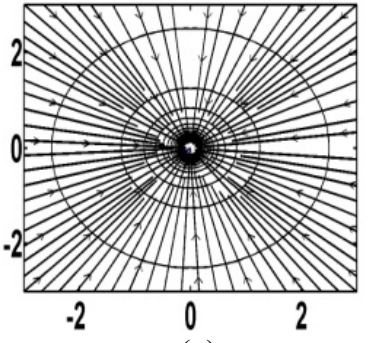

(a)

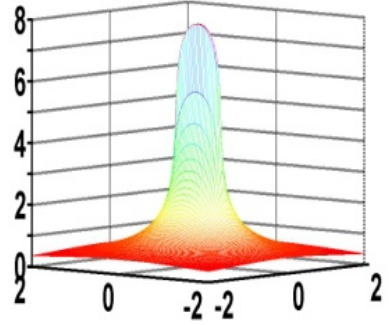

(b)

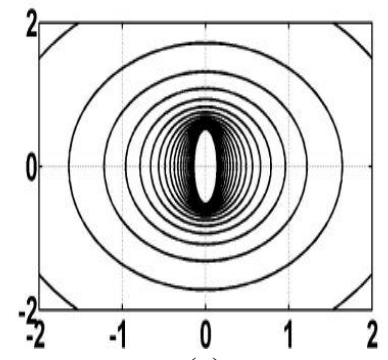

(c)

Figure 1. Distribution of equivalent surface and electrical potential of common uniformly charged regular bodies. (a) is for the spherical surface; (b) and (c) are for the long straight wire.

\section{Distributions of Potential Surface between Different Charged Objects}

\section{Electric Dipole}

Electric dipole is a system consisting of two positive and negative charges with same electricity. Using the superposition principle, the expression of electric potential is obtained as follows:

$$
U=\frac{q}{4 \pi \varepsilon_{0} \sqrt{\left(x-a_{1}\right)^{2}+\left(y-b_{1}\right)^{2}+\left(z-c_{1}\right)^{2}}}+\frac{q}{4 \pi \varepsilon_{0} \sqrt{\left(x-a_{2}\right)^{2}+\left(y-b_{2}\right)^{2}+\left(z-c_{2}\right)^{2}}} .
$$

Where $\left(a_{1}, b_{1}, c_{1}\right)$ and $\left(a_{2}, b_{2}, c_{2}\right)$ are the location of the positive and negative charges respectively. Then the corresponding potential and equipotential surface distribution are drawn as shown in Fig. 2(a) and (b).

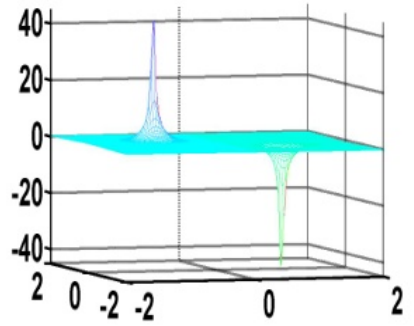

(a)

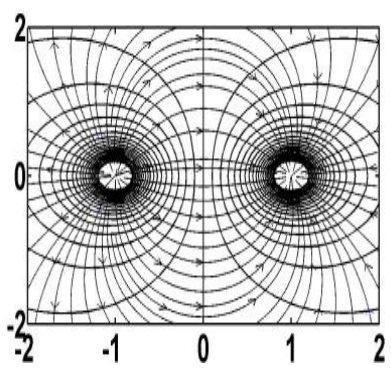

(b)

Figure 2. The potential and equipotential surface distributions of the electric dipole in (a) and (b) separately.

In order to facilitate simulation, here we set one of the three-dimensional coordinate axes as a constant. According to Eq. 3 and Fig. 2(a), the absolute value of the potential decreases with the 
increase of the other two coordinates. Fig. 2(b) shows that the equipotential surfaces are around positive and negative charges separately, and their shapes are similar to ellipses. The distributions of the equipotential surface near the two charges are dense and slightly deformed, which indicates that the electric field is strong here.

\section{Two Charged Conductor Spheres}

According to the previous analysis, the combination of two conductor spheres, i.e., sphere surface is similar with the electric dipole system. The coordinates of the two sphere center are $(a, b, c)$ and $(l, m$, $n$ ) respectively, and the charged quantity is q. According to the principle of superposition, the expression of potential can be written as follows:

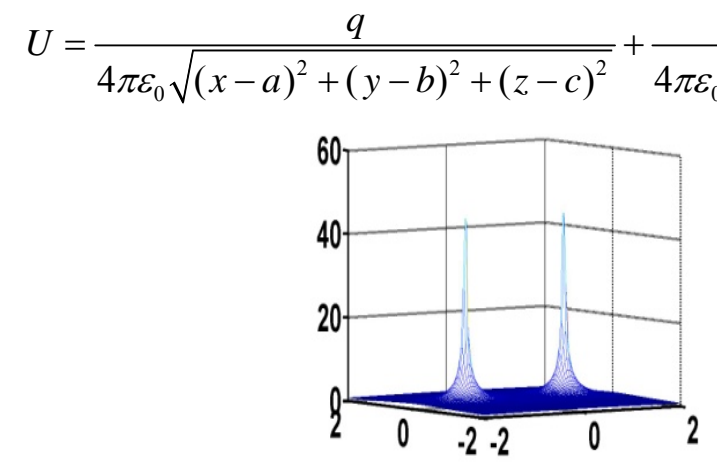

(a)

Figure 3. The potential and equipotential surface distributions of two charged conductor spheres in (a) and (b) separately.

Note that the coordinate $(x, y, z)$ of the investigation point in Eq. 4 is out of the two spheres. The potential distribution and equipotential surface are shown as Fig. 3(a) and (b). The effect of charge distribution on the equipotential surface can be seen from the figure. Near the sphere surface, the electric field is strong, and the equipotential surfaces are concentrated surrounding each spheres. In the process of moving away from the sphere, the equipotential surfaces of the two spheres begin connected and gradually become ellipsoids surrounding both two spheres. Because the electric potential distribution of the charged sphere surface is similar to the point charge, comparing Fig. 3(b) with Fig. 2(b), one can find that the equipotential surface of the electric dipole concentrates on the two charges and are basically elliptical. Here both of the conductor spheres have identical charge, so the equipotential surface surrounds the two sphere surface and gradually transits from dumbbell to ellipse shape. This indicates that the distribution of equipotential surface is also affected by the charge property.

\section{Long Straight Wire and Single Point Charge}

On the basis of the previous work, we can also find out the relationship between the potential of a single point charge and a long straight wire. By using the same treatment method, the principle of potential superposition, we can get the expression of the potential of the system as follows:

$$
U=\frac{\lambda}{4 \pi \varepsilon_{0}} \ln \frac{z+\frac{l}{2}+\sqrt{r^{2}+\left(z+\frac{l}{2}\right)^{2}}}{z-\frac{l}{2}+\sqrt{r^{2}+\left(z-\frac{l}{2}\right)^{2}}}+\frac{q}{4 \pi \varepsilon_{0} \sqrt{(x-a)^{2}+(y-b)^{2}+(z-c)^{2}}} .
$$

By simplifying the parameters in Eq. 5, the potential distribution and equipotential surface maps are shown in Fig. 4(a) and (b). Although only part of the equipotential lines can be drawn in Fig. 4(b) for space limitation, it can show that the effects of charge distribution and the shape of charged body on the distribution of electric potential and equipotential surface. 


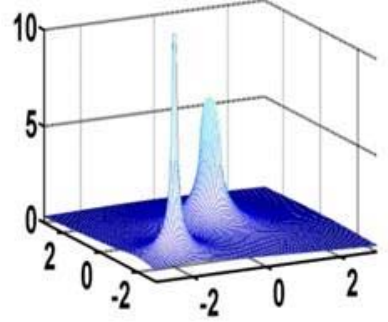

(a)

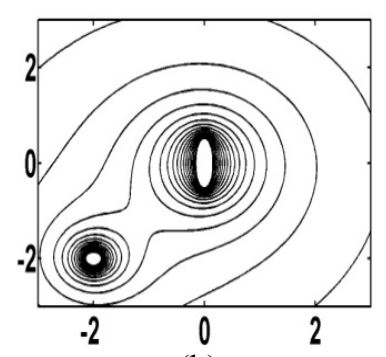

(b)

Figure 4. The potential and equipotential surface distributions of the combination of the long straight wire and single point charge in (a) and (b) separately.

\section{Electric Dipole and Long Straight Wire}

In fact, the combination of electric dipole and long straight wire can be seen as two point charges and long straight wire. According to the principle of superposition, the potential expression of the combination is written as follows:

$$
U=\frac{\lambda}{4 \pi \varepsilon_{0}} \ln \frac{z+\frac{l}{2}+\sqrt{r^{2}+\left(z+\frac{l}{2}\right)^{2}}}{z-\frac{l}{2}+\sqrt{r^{2}+\left(z-\frac{l}{2}\right)^{2}}}+\frac{q}{4 \pi \varepsilon_{0} \sqrt{(x-a)^{2}+(y-b)^{2}+(z-c)^{2}}}-\frac{q}{4 \pi \varepsilon_{0} \sqrt{(x-l)^{2}+(y-m)^{2}+(z-n)^{2}}} .
$$

After setting the specific parameters, the potential distribution and equipotential surfaces are drawn in Fig. 5(a) and (b). It can be seen that the equipotential surface has the characteristics of dense equipotential surface and sparse equipotential surface where the electric field is strong or weak. Comparing Fig.(i) with Fig.(e), the difference between the two combinations is that one is composed of electric dipole, the other is composed of electric dipole and long straight wire. But the distribution of the equipotential surface of the two combinations is different, which shows that the shape of charged body has an influence on the distribution of electric potential and equipotential surface.

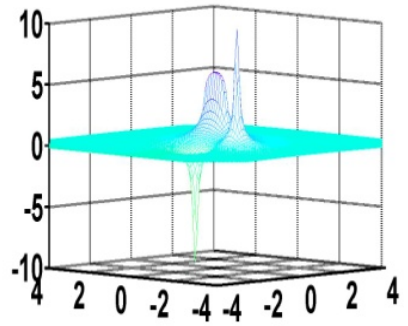

(a)

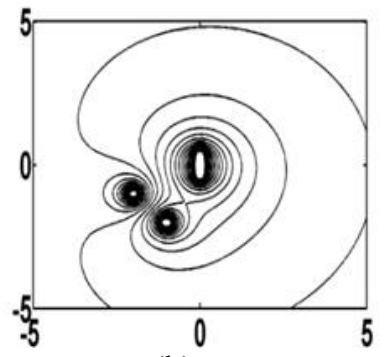

(b)

Figure 5. The potential and equipotential surface distributions of the combination of the electric dipole and long straight wire in (a) and (b) separately.

\section{Combination of Five Point Charges}

In Cartesian coordinate system, the coordinates of individual point charges are set as $(-5,-5,0)$, and the other coordinates of four point charges forming square are $(1,1,0),(1,-1,0),(-1,1,0),(-1,-1,0)$. Using the principle of superposition, the expression of total potential is obtained as follows:

$$
U=\frac{q}{4 \pi \varepsilon_{0}}\left[\frac{1}{\sqrt{(x+5)^{2}+(y+5)^{2}}}+\frac{1}{\sqrt{(x-1)^{2}+(y-1)^{2}}}+\frac{1}{\sqrt{(x-1)^{2}+(y+1)^{2}}}+\frac{1}{\sqrt{(x+1)^{2}+(y-1)^{2}}}+\frac{1}{\sqrt{(x+1)^{2}+(y+1)^{2}}}\right] \cdot(7)
$$

The potential and equipotential surface distribution maps are drawn in Fig. 6(a) and (b). It can be seen that since the point charge $\mathrm{q} 1$ is relatively far away from the other four point charges (q2, $\mathrm{q} 3$, $\mathrm{q} 4$,q5), the equipotential surface near q1 is spherical, while the other four charges equipotential surfaces are approximately spherical around them separately. In the space far from all the five charges in the meantime, the total equipotential surface begins to appear ellipsoidal around the 
combination. Comparing Fig. 3(b) with Fig. 6(b), we can find that although the two combinations are both composed by positive charges, the potential distribution and equipotential surface shape are quite different, which indicates that the number of charges have strong effects on them.

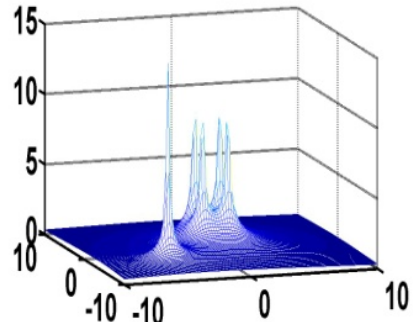

(a)

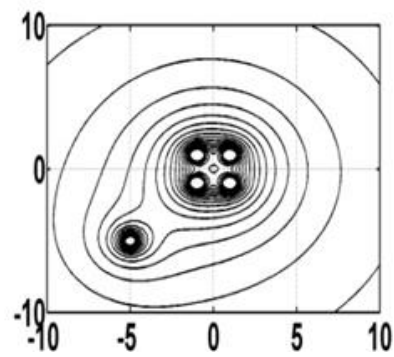

(b)

Figure 6. The potential and equipotential surface distributions of the five point charges combination in (a) and (b) separately.

\section{Summary}

By using the relevant theorems of Electromagnetics, the expressions of electric potential of several uniformly charged regular bodies are given, and the equipotential surfaces distributions of charged body combination are drawn by MATLAB, of which the characteristics and change rules are analyzed. It is concluded that the types of charges, the shapes of charged bodies (charge distribution) and the position of charged bodies in the 3D space all have influence on the distributions of electric potential and equipotential surface.

According to the above work, a feasible method is provided when the charged bodies for some specific electric fields are need to be designed. That is, the shape and charge distribution of the charged bodies can be designed in advance, and then the corresponding potential distribution can be simulated by the software like MATLAB and compared with the original requirements for improvement further.

\section{Acknowledgement}

This work is supported by the following funds: Quality Course Construction of Colleges and Universities in Shandong Province (JPKC201311), Quality Course Construction of Shandong Province Graduate Education (1907319).

\section{References}

[1] Q.S. Ji, H.Y. Hao, C.X Zhang, et al. Physical energy gap and Landau level in silicone controlled by electric field, Journal of Physics. 8 (2015) 313-321.

[2] U. Wu, J. Xiao, J. Wu, et al. Laser radiation spectra of dye-doped chiral nematic liquid crystal devices under electric field, Spectroscopy \& spectral analysis. 5 (2016) 1313-1316.

[3] J.Z. Han and Q. Xing. Thermodynamic mechanism of dielectric electrothermal effect under electrostatic field, Journal of South China University of Technology(Natural Science Edition). 6 (2017) 20-24.

[4] K. Chen and X.M. Yi. Examples of common solutions to electric field intensity in competitions, Physics of Hunan Middle School. 8 (2011) 77-78. 